Article

\title{
Indole-3-Acetic Acid Biosynthesis Pathways in the Plant-Beneficial Bacterium Arthrobacter pascens ZZ21
}

\author{
Mengsha Li ${ }^{1}$, Rui Guo ${ }^{1}$, Fei Yu ${ }^{1}$, Xu Chen ${ }^{1}$, Haiyan Zhao ${ }^{2}$, Huixin $\mathrm{Li}^{1}{ }^{1, *}$ and Jun $\mathrm{Wu}^{1, *}$ \\ 1 Soil Ecology Lab, College of Resources and Environmental Science, Nanjing Agricultural University, \\ Nanjing 210095, China; 2014203018@njau.edu.cn (M.L.); 2015203007@njau.edu.cn (R.G.); \\ 2014203007@njau.edu.cn (F.Y.); 2015203006@njau.edu.cn (X.C.) \\ 2 College of Resources and Environmental Science, Nanjing Agricultural University, Nanjing 210095, \\ China; haiyanzhao@njau.edu.cn \\ * Correspondence: huixinli@njau.edu.cn (H.L.); wujun2013@njau.edu.cn (J.W.); Tel.: +86-025-8439-5374 (H.L.)
}

Received: 30 December 2017; Accepted: 30 January 2018; Published: 1 February 2018

\begin{abstract}
Arthrobacter pascens ZZ21 is a plant-beneficial, fluoranthene-degrading bacterial strain found in the rhizosphere. The production of the phytohormone indole-3-aectic acid (IAA) by ZZ21 is thought to contribute to its ability to promote plant growth and remediate fluoranthene-contaminated soil. Using genome-wide analysis combined with metabolomic and high-performance liquid chromatography-mass spectrometry (HPLC-MS) analyses, we characterized the potential IAA biosynthesis pathways in $A$. pascens ZZ21. IAA production increased 4.5 -fold in the presence of $200 \mathrm{mg} \cdot \mathrm{L}^{-1}$ tryptophan in the culture medium. The transcript levels of prr and ald $H$, genes which were predicted to encode aldehyde dehydrogenases, were significantly upregulated in response to exogenous tryptophan. Additionally, metabolomic analysis identified the intermediates indole-3-acetamide (IAM), indole-3-pyruvic acid (IPyA), and the enzymatic reduction product of the latter, indole-3-lactic acid (ILA), among the metabolites of ZZ21, and subsequently also IAM, ILA, and indole-3-ethanol (TOL), which is the enzymatic reduction product of indole-3-acetaldehyde, by HPLC-MS. These results suggest that the tryptophan-dependent IAM and IPyA pathways function in ZZ21.
\end{abstract}

Keywords: Arthrobacter; IAA biosynthesis pathway; genome-wide analysis; metabolomics; HPLC-MS

\section{Introduction}

Indole-3-acetic acid (IAA), the most common naturally occurring auxin, is a hormone produced by plants, fungi and bacteria. IAA plays a central role in modulating plant growth and development [1-3]. In addition to being produced by plants, IAA is produced by some beneficial bacteria in the rhizosphere, where it acts as a signaling molecule that has significant effects on the communication between plants and microorganisms and promotes plant growth [4-6]. Tryptophan (Trp) is a main precursor for IAA biosynthesis in microbes [5]. Based on the distinct intermediates involved in tryptophan-dependent IAA biosynthesis, five different pathways have been characterized in bacteria: the indole-3-acetamide (IAM), indole-3-pyruvic acid (IPyA), indole-3-acetonitrile (IAN), tryptamine (TAM), and tryptophan side-chain oxidase (TSO) pathways [7,8]. Although the tryptophan-independent pathway is also thought to occur in bacteria [9], no specific enzymes in this pathway have been characterized.

Pathways and key genes involved in IAA biosynthesis in Gram-negative bacteria are well documented, but reports on the biosynthesis pathways used by Gram-positive bacteria are lacking. The Gram-positive, spore-forming bacterium Paenibacillus polymyxa E681 was shown to synthesize IAA via the IPyA pathway, and the key gene ipdC (encoding indole-3-pyruvate decarboxylase) was 
functionally identified and found to be constitutively expressed [10]. The main biosynthetic route used by the phytopathogen Rhodococcus fascians is the IPyA pathway [11]. The IAN, TAM and IPyA pathways and an uncharacterized pathway are thought to function in Bacillus amyloliquefaciens SQR9. The genes patB (encoding a conserved hypothetical protein predicted to be an aminotransferase), $y c l C$ (encoding a UbiD family decarboxylase) and dhaS (encoding indole 3-acetaldehyde dehydrogenase) constitute a putative complete IPyA pathway that mediates the biosynthesis of IAA in this bacterium [12]. Arthrobacter, one of the largest groups of soil bacteria, produces IAA when tryptophan is present in the medium [13-16]. An early study by Mino demonstrated that the IPyA and IAM pathways function in Arthrobacter spp. [17]. However, the details of IAA biosynthesis that function in Arthrobacter still require further study.

Arthrobacter pascens ZZ21 was isolated from the rhizosphere of forest soil from Zijin Mountain in Nanjing City, Jiangsu Province, China. It is a multifunctional, salt-tolerant bacterial strain that secretes IAA, dissolves inorganic phosphorus and degrades fluoranthene, which belongs to the polycyclic aromatic hydrocarbons (PAHs) class of persistent organic pollutants (POPs). IAA production in A. pascens ZZ21 can reach a level of $92.31 \mathrm{mg} \cdot \mathrm{L}^{-1}$ under optimal conditions ( $\mathrm{pH} 6$, liquid volume of $45 \mathrm{~mL}$ medium in a $150 \mathrm{~mL}$ conical flask, temperature of $28^{\circ} \mathrm{C}$, shaking time of $15 \mathrm{~h}$, and mannitol and yeast extract as carbon and nitrogen sources respectively) [18]. We previously reported that the IAA produced by $A$. pascens ZZ21 increases soil microbial activities and contributes to its plant-growth-promoting effects. We also demonstrated that the application of IAA-producing A. pascens ZZ21 combined with the fluoranthene-degrading strain Bacillus cereus Z21 is more effective at remediating fluoranthene-contaminated soil than ZZ21 alone [19]. IAA produced by A. pascens ZZ21 promotes plant growth and enhances tolerance to fluoranthene, leading to increased fluoranthene uptake by plants, thereby improving a plant's ability to remediate fluoranthene-contaminated soils.

In the current study, using a combination of genetic and chemical analyses, we characterized potential IAA biosynthesis pathways in A. pascens ZZ21. We identified candidate genes involved in IAA biosynthesis through genome-wide screening and examined their transcriptional responses to exogenous tryptophan. Finally, we detected potential intermediates involved in possible IAA biosynthesis pathways using metabolomic analysis and HPLC-MS, thereby shedding light on the regulatory mechanism underlying the soil-remediation and plant-growth-promoting effects of this bacterium.

\section{Results}

\subsection{Whole-Genome Sequencing of A. pascens ZZ21 and Screening for Genes Likely Involved in} IAA Biosynthesis

We assembled the entire $A$. pascens ZZ21 genome and mined it for genes involved in IAA metabolism. Sequencing data statistics are listed in Table S1. Statistical parameters used for the genome assembly are indicated in Table S2, and gene information statistics are listed in Table S3. Based on the reported genes and IAA biosynthesis pathways [4,8,20], we performed a Blast analysis of the predicted ZZ21 genes against the Kyoto Encyclopedia of Genes and Genomes (KEGG) and National Center for Biotechnology Information (NCBI) databases using their deduced protein sequences as queries and identified candidate genes involved in tryptophan-dependent IAA biosynthesis (Figure S1; Table 1). For the IAM pathway, comparative sequence analyses showed that the deduced protein sequence of iaaM (orf0652) in ZZ21 had obviously sequence similarity to characterized tryptophan 2-monoxygenase, sharing at $88 \%$ identification with tryptophan 2-monxygenase from Arthrobacter sp. P2b (GenBank accession No. SLJ94339.1) and 89\% identification with tryptophan 2-monxygenase from Arthrobacter sp. ok362 (GenBank accession No. SDK38081.1). The deduced protein sequences of genes aam (orf3469) and gat $A$ (orf0389) shared 90\% identification with amidase from Arthrobacter sp. Soil736 (NCBI Reference Sequence No. WP_056629692.1) and $81 \%$ identification with amidase from Arthrobacter globiformis (NCBI Reference Sequence No. WP_087872787.1), respectively. We identified the iaaM-homologous gene, which was predicted 
to encode tryptophan 2-monooxygenase, and the amidase genes (aam, gatA), which may function in the IAM hydrolysis reaction (Table 1). The final step in the IPyA, TAM, and TSO pathways is the oxidative dehydrogenation of indole-3-acetaldehyde (IAAld) to IAA, which is catalyzed by aldehyde dehydrogenase. Comparative sequence analyses showed that the deduced protein sequences of prr (orf1423), puuC (orf2422) and aldH (orf3473) had an obvious overall sequence similarity to characterized aldehyde dehydrogenase, respectively sharing $96 \%$ identification with aldehyde dehydrogenase from Arthrobacter nitrophenolicus (GenBank accession No. ELT45240.1), 94\% identification with aldehyde dehydrogenase from Arthrobacter globiformis (NCBI Reference Sequence No. WP_003803492.1) and 93\% identification with aldehyde dehydrogenase from Arthrobacter sp. 35W (NCBI Reference Sequence No. WP_026555119.1). Thus, prr, puuC, and aldH might encode this enzyme in ZZ21. However, the putative amino transferase gene and the well-documented indole-3-pyruvate decarboxylase ipdC gene in the IPyA pathway, as well as genes encoding the putative amine oxidase and tryptophan decarboxylase in the TAM pathway, were not detected in the ZZ21 genome. Finally, no genes involved in the IAN pathway were found in the ZZ21 genome.

Table 1. Identity of proteins encoded by possible genes involved in IAA ${ }^{\text {a }}$ biosynthesis in ZZ21.

\begin{tabular}{|c|c|c|c|c|}
\hline $\begin{array}{c}\text { IAA } \\
\text { Biosynthesis Pathways }\end{array}$ & ZZ21 GID & $\begin{array}{l}\text { Products and Entry Numbers } \\
\text { in KEGG }\end{array}$ & NCBI Refseq or GenBank & Identity (\%) \\
\hline \multirow{3}{*}{ IAM } & iaaM (orf0652) & $\begin{array}{c}\text { tryptophan 2-monooxygenase } \\
\text { (EC 1.13.12.3) }\end{array}$ & $\begin{array}{c}\text { SLJ94339.1 } \\
\text { (Arthrobacter sp. P2b) }\end{array}$ & 88 \\
\hline & aam (orf3469) & $\begin{array}{c}\text { amidase } \\
(\mathrm{EC} 3.5 .1 .4)\end{array}$ & $\begin{array}{c}\text { WP_056629692.1 } \\
\text { (Arthrobacter sp. Soil736) }\end{array}$ & 90 \\
\hline & gat A (orf0389) & $\begin{array}{c}\text { amidase } \\
(\mathrm{EC} 3.5 .1 .4)\end{array}$ & $\begin{array}{c}\text { WP_087872787.1 } \\
\text { (Arthrobacter globiformis) }\end{array}$ & 81 \\
\hline \multirow{3}{*}{ IPyA/TAM/TSO } & prr (orf1423) & $\begin{array}{c}\text { aldehyde dehydrogenase } \\
\text { (EC 1.2.1.3) }\end{array}$ & $\begin{array}{c}\text { ELT45240.1 } \\
\text { (Arthrobacter nitrophenolicus) }\end{array}$ & 96 \\
\hline & puuC (orf2422) & $\begin{array}{l}\text { aldehyde dehydrogenase } \\
\text { (EC 1.2.1.3) }\end{array}$ & $\begin{array}{c}\text { WP_003803492.1 } \\
\text { (Arthrobacter globiformis) }\end{array}$ & 94 \\
\hline & aldH (orf3473) & $\begin{array}{c}\text { aldehyde dehydrogenase } \\
\text { (EC 1.2.1.3) }\end{array}$ & $\begin{array}{c}\text { WP_026555119.1 } \\
\text { (Arthrobacter sp. } 35 \mathrm{~W})\end{array}$ & 93 \\
\hline
\end{tabular}

${ }^{a}$ IAA: indole-3-acetic acid.

2.2. Identification of Candidate Genes Involved in Tryptophan-Dependent IAA Biosynthesis in A. pascens ZZ21 Based on Their Transcriptional Responses to Tryptophan

Since the candidate genes identified by genome-wide sequencing are likely involved in tryptophan-dependent IAA biosynthesis, we reviewed their transcriptional response to exogenous tryptophan in the culture medium. IAA production in ZZ21 increased 4.5 -fold when $200 \mathrm{mg} \cdot \mathrm{L}^{-1}$ L-tryptophan was added to the Luria-Bertani (LB) medium (Figure 1a), indicating that IAA biosynthesis in ZZ21 is significantly induced by tryptophan. Among the six candidate genes, only two were significantly induced by exogenous tryptophan (Figure $1 \mathrm{~b}$ ). The expression of prr and aldH, which were predicted to encode aldehyde dehydrogenases, increased 2.8-fold and 1.8-fold, respectively, in response to exogenous tryptophan. These two genes were annotated and predicted to participate in the final steps of the tryptophan-dependent IPyA, TAM and TSO IAA biosynthesis pathways. The expression of the four other candidate genes did not significantly change in response to exogenous tryptophan treatment, suggesting that these four genes were insensitive to exogenous tryptophan or did not actually function in IAA biosynthesis in ZZ21 (Figure 1b). 

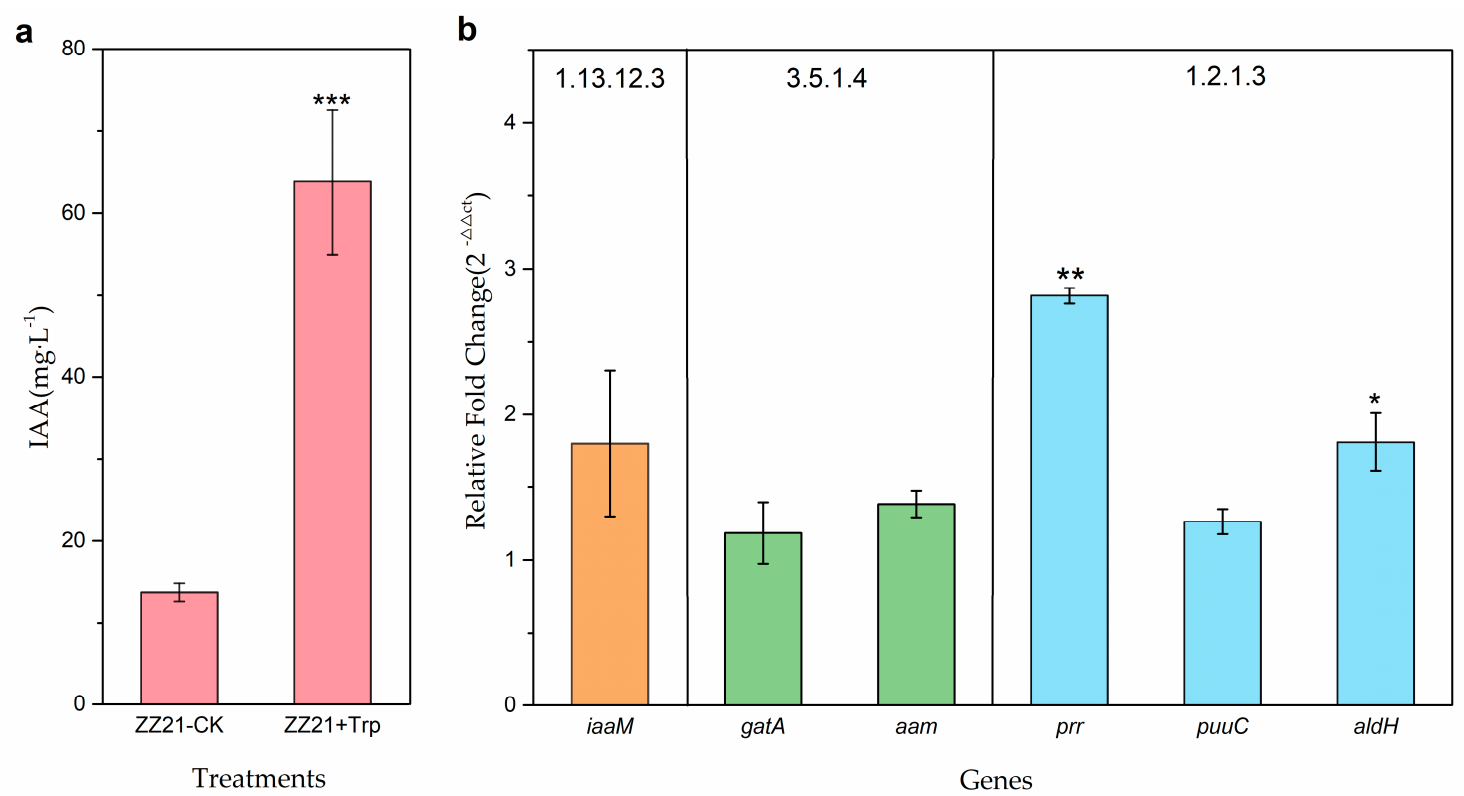

Figure 1. IAA production and genes transcript levels in $Z Z 21$ in response to tryptophan. (a) IAA production in A. pascens ZZ21 cultured for $48 \mathrm{~h}$ with or without $200 \mathrm{mg} \cdot \mathrm{L}^{-1} \mathrm{~L}$-tryptophan in Luria-Bertani (LB) medium. (b) Relative expression of genes in ZZ21 cultured in the absence or presence of tryptophan (Trp). Gene transcript levels were evaluated by qRT-PCR. ZZ21 was grown in LB medium with or without tryptophan for $48 \mathrm{~h}$. The 16sRNA gene of ZZ21 was used as an internal reference. Error bars represent standard errors of three biological replicates. One-way analysis of variance (ANOVA) was performed. ${ }^{*} p<0.05,{ }^{* *} p<0.01,{ }^{* * *} p<0.001$.

\subsection{Detection of Intermediates in the IAA Biosynthesis Pathway in A. pascens ZZ21 by Metabolomic Analysis}

We performed a metabolomic analysis to measure the levels of IAA biosynthesis intermediates in A. pascens ZZ21. We visualized the total ion current (TIC) of the A. pascens ZZ21 samples, which exhibited strong signals, a large peak capacity, and good reproducibility of retention times (Figure S2). Peak alignment of all raw data revealed 725 possible metabolites. After all internal standards and any known pseudo-positive peaks caused by background noise, column bleed, or the BSTFA ( $\mathrm{N}, \mathrm{O}$-Bis(trimethylsilyl)trifluoroacetamide) derivatization procedure, were removed, a total of 213 metabolites were detected.

Based on the proposed IAA biosynthesis pathways in bacteria $[4,8,20]$, we mined the metabolites of A. pascens ZZ21 for intermediates involved in each step of these pathways. Indole-3-pyruvic acid and indole-3-acetamide were detected among the metabolites of ZZ21 (Figure 2). The response intensity of IPyA was much lower than that of IAM, and IAAld was not detected among the metabolites of ZZ21, as IPyA and IAAld are unstable and accumulate at almost undetectable levels in culture $[7,21]$. Indole-3-lactic acid (ILA), the product of the enzymatic reduction of IPyA, was detected in ZZ21, suggesting that IAA biosynthesis in ZZ21 involves the IPyA pathway (Figure 2). 


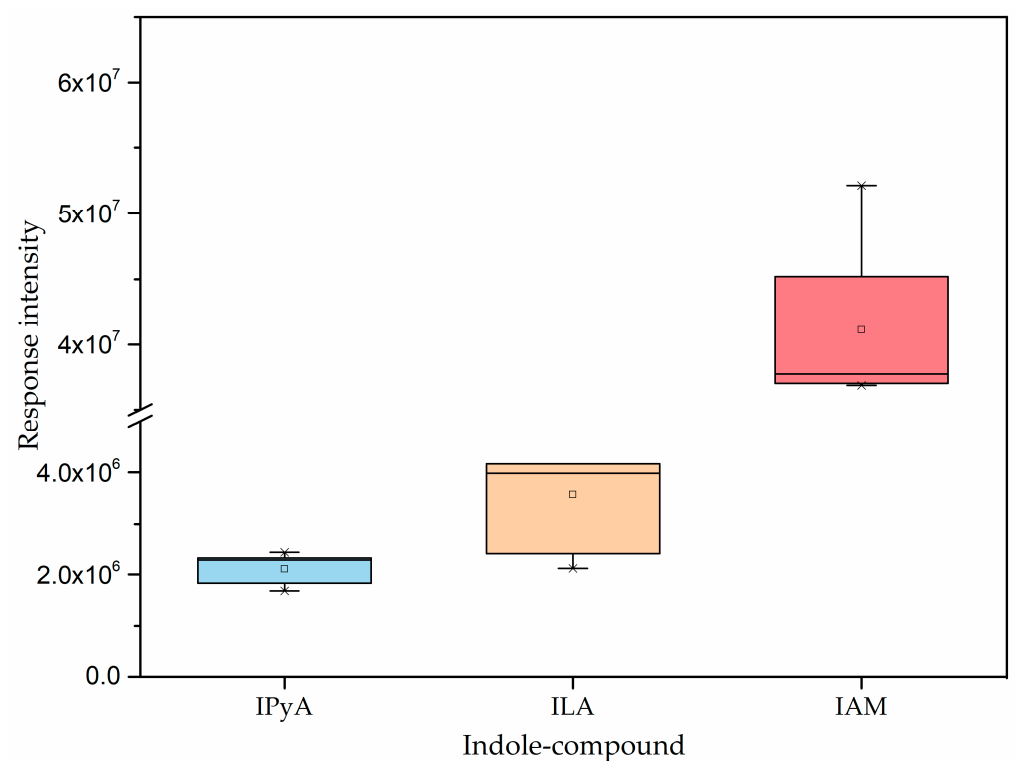

Figure 2. Indole derivatives detected among ZZ21 metabolites by metabolomic analysis. Bars represent standard deviations of five biological replicates. IPyA: indole-3-pyruvic acid; ILA: indole-3-lactic acid; IAM: indole-3-acetamide.

\subsection{Identification of Candidate Intermediates Involved in IAA Biosynthesis in A. pascens ZZ21 by HPLC-MS}

Based on the results of the genome-wide sequencing and metabolomic analysis, we identified two possible IAA biosynthesis pathways in A. pascens ZZ21. To clarify the potential IAA biosynthesis pathway used by ZZ21, we further identified IAM (the key intermediate in the IAM pathway), ILA (the product of enzymatic reduction of IAAld in the IPyA pathway) and indole-3-ethanol (TOL) (an IAAld catabolic product) by HPLC-MS. The retention time of IAA, IAM, TOL and ILA in ZZ21 metabolites were similar to their standards. After hydrogenation, the relative molecular masses of the four indole compounds were 176.1, 175.2, 206.1, and 162.2, respectively (Figure 3A). IAM and TOL were detected in ZZ21 (Figure 3b,c), whereas no defined peak was detected for ILA (Figure 3d). Therefore, we used secondary ion mass spectrometry in an effort to identify ILA by detecting two characteristic ILA fragment peaks (Figure 3B). Two peaks at $118.1\left(206.1-88=\mathrm{C}_{8} \mathrm{H}_{7} \mathrm{~N}\right) \mathrm{m} / \mathrm{z}$ and $130.1\left(206.1-88=\mathrm{C}_{9} \mathrm{H}_{8} \mathrm{~N}\right) \mathrm{m} / z$, which are characteristic masses identified in ILA fragmentation patterns, were detected in ZZ21 metabolic samples (Figure 3f,g). The concentration of TOL was significantly increased when exogenous tryptophan was supplied to the medium (Figure 4). These results indicated that ILA is indeed present in ZZ21. Taken together, the IAM and IPyA pathways are thought to function in ZZ21. 


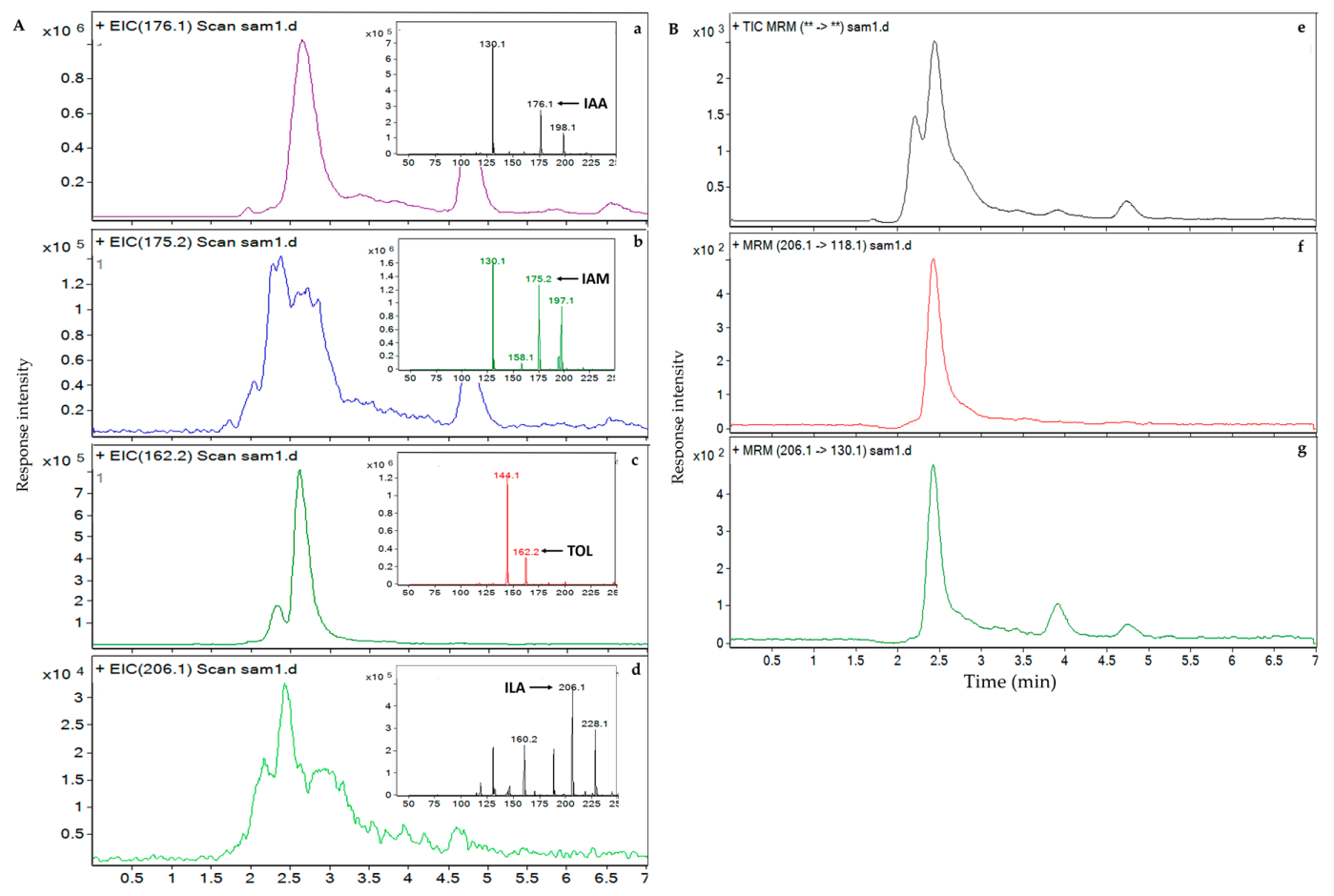

Figure 3. High-performance liquid chromatography-mass spectrometry (HPLC-MS) analysis of the four target indole compounds in ZZ21. (A) Extracted ion chromatography (EIC) of the four indole compounds involved in IAA biosynthesis in ZZ21: (a) IAA: indole-3-acetic acid, (b) IAM: indole-3-acetamide, (c) ILA: indole-3-lactic acid, and (d) TOL: indole-3-ethanol. The multiple reaction monitoring (MRM) chromatography of the four indole-compounds were showed on the top right part of each EIC figure. The fragmentor voltage was $135 \mathrm{~V}$. (B) MRM chromatography of the characteristic fragments of indole-3-lactic acid in ZZ21 (e). $118.1 \mathrm{~m} / \mathrm{z}(\mathbf{f})$ and $130.1 \mathrm{~m} / \mathrm{z}(\mathbf{g})$ are two characteristic peaks of indole-3-lactic acid fragments. The fragmentor voltage was $90 \mathrm{~V}$.

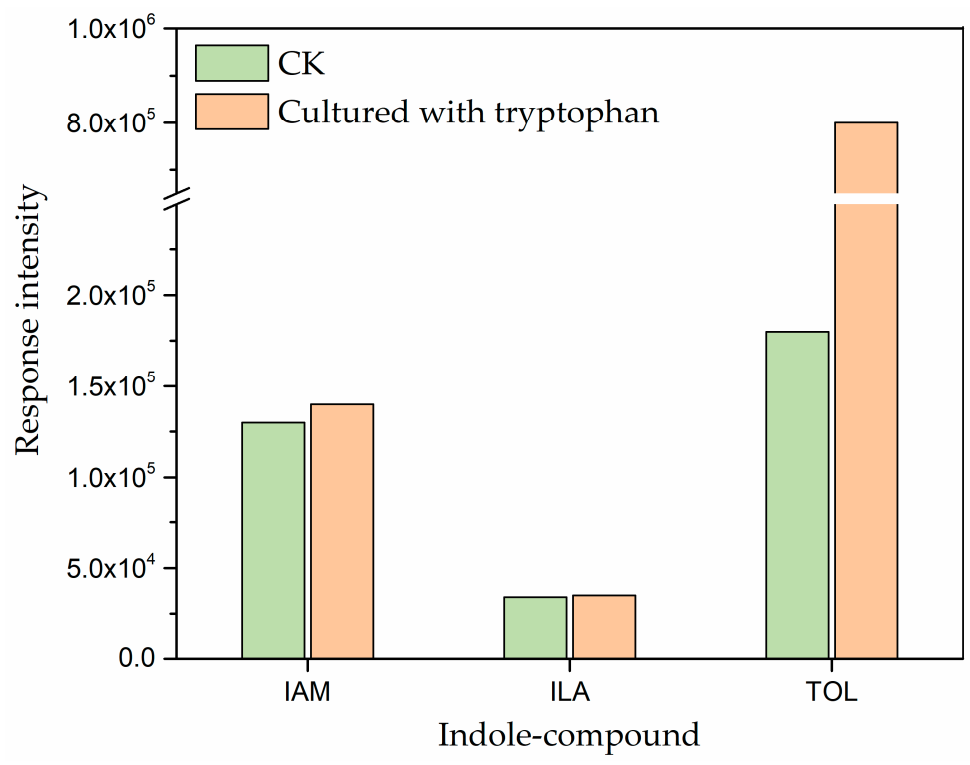

Figure 4. Analysis of the intermediates in ZZ21 grown in the medium with or without (CK: control check) tryptophan by HPLC-MS. IAM: indole-3-acetamide; ILA: indole-3-lactic acid; TOL: indole-3-ethanol. 


\section{Discussion}

IAA biosynthesis in various Arthrobacter species (A. crystallopoietes, A. globiformis, A. nicotianae, A. sulfonivorans, and A. sulfureus) is stimulated by the application of exogenous tryptophan [13-15]. Similarly, in the current study, we detected a significant increase in IAA production after the addition of tryptophan to the ZZ21 culture medium, indicating that IAA biosynthesis in ZZ21 is induced by tryptophan (Figure 1a). To identify possible IAA biosynthesis pathways in ZZ21, we used three different methods involving combined genetic and chemical analyses.

We found that the IAM, IPyA, TAM and TSO pathways likely exist in ZZ21, as six possible functional genes in these pathways were detected via gene prediction and annotation (Figure S1; Table 1). The IAM pathway is the best-characterized pathway in bacteria [22,23]. In this two-step pathway, tryptophan is catalyzed to the intermediate IAM, which is then converted to IAA (Figure S1 and Figure 5) [4,8]. The main genes driving the IAM pathway are iaaM and iaaH, encoding tryptophan 2-monooxygenase and a specific IAM hydrolase/amidase, respectively [23-25]. We detected iaaM-homologous gene in the ZZ21 genome, as well as aam and gat $A$, which were predicted to encode amidases (Table 1). Amidase, which functions in the second step of the IAM pathway, has been purified from the Arthrobacter sp. strain J-1 [26]. However, iaaM, aam and gatA showed no significant changes in transcript levels in response to tryptophan and it is therefore unclear how much the IAM pathway contributes to IAA production in ZZ21. An early study in P. syringae pv. savastanoi demonstrated that IAA production dramatically increases in response to exogenously supplied tryptophan without significantly altering tryptophan monooxygenase activity [27] and the transcription of iaaM and iaaH. Similarly, Gaffney et al. demonstrated that the expression of these genes in the IAM pathway is continuous and does not require an exogenous supply of tryptophan [28]. However, tryptophan 2-monooxygenase encoded by iaaM is negatively regulated by both IAM and IAA [29]. Therefore, the genes involved in the IAM pathway are possibly insensitive to exogenous tryptophan but sensitive to feedback inhibition by its reaction products. Taken together, the existence of the IAM pathway in ZZ21 remains to be confirmed.

The IPyA pathway is thought to function in both plants and bacteria, including Azospirillum, Enterobacter, Rhizobium, and Bradyrhizobium [4]. In the first step of this pathway, tryptophan is converted to IPyA by an aminotransferase [30,31]. The conversion of IPyA to IAAld by indole-3-pyruvate decarboxylase (IPDC) (encoded by $i p d C$ ) is the rate-limiting step in this process [32-35]. IAAld is then oxidized to IAA by aldehyde dehydrogenase, mutase, or oxidase enzymes (Figure S1 and Figure 5) [36,37]. In Bacillus amyloliquefaciens, dhaS is thought to encode indole-3-acetaldehyde dehydrogenase [12,38]. The TAM pathway was identified in Azospirillum based on the conversion of exogenous TAM to IAA (Figure S1 and Figure 5) [39] and in Bacillus cereus based on the identification of tryptophan decarboxylase activity [40]. Nevertheless, the bacterial genes encoding related enzymes in this pathway must still be confirmed.

To date, the TSO pathway has only been demonstrated in Pseudomonas fluorescens CHA0. In this pathway, tryptophan is converted to IAAld and then oxidized to IAA (Figure S1 and Figure 5) [41]. In the ZZ21 genome, we identified $p r r$, puuC, and ald $H$, which were predicted to encode aldehyde dehydrogenases involved in the final steps of the IPyA, TAM and TSO pathways. These three genes were found to encode aldehyde dehydrogenases in Pseudomonas putida KT2440 [42], Escherichia coli K-12 [43], and Bifidobacterium bifidum PRL2010 [44], respectively, but a recent study on vermicompost-borne bacteria suggested that ald $H$ is likely not specifically involved in IAA biosynthesis [45]. While some aldehyde dehydrogenases show high specificity for a few substrates, others exhibit low specificity for a wide range of substrates [8]. Aldehyde dehydrogenase requires $\mathrm{NAD+}$ or NADP+ as an electron acceptor to convert IAAld to IAA [46-48]. In the current study, quantitative reverse-transcription PCR (qRT-PCR) analysis indicated that prr and aldH were significantly upregulated in ZZ21 in response to tryptophan (Figure 1b). This result suggests that these two genes are thought to be involved in IAA biosynthesis in ZZ21 and puuC is not. However, neither the well-documented indolepyruvate decarboxylase ipdC gene in the IPyA pathway 
nor the tryptophan side chain oxidase/tryptophan transaminase genes in the TSO pathway were detected in ZZ21, nor were genes involved in the TAM pathway. Consequently, we were unable to determine which of these pathways is used by ZZ21 to synthesize IAA. The IAN pathway is not as well characterized in bacteria as it is in plants. The microbial enzymes responsible for the initial conversion of tryptophan to indole-3-acetaldoxime have not been detected in bacteria. Indole-3-acetaldoxime is subsequently converted to IAN by an indoleacetaldoxime dehydratase (encoded by oxd genes), which has been identified in several bacteria [49]. Finally, IAN is transformed into IAA by a nitrilase in a single step [50] or by a nitrile hydratase and an amidase in two steps (Figure S1 and Figure 5) [51]. In the current study, nitrile hydratase and nitrilase, which were reported in Arthrobacter [26,52,53], were not detected in the ZZ21 genome. To determine the pathway(s) used by ZZ21 to synthesize IAA, chemical methods might be needed to detect intermediates involved in various IAA biosynthesis pathways.

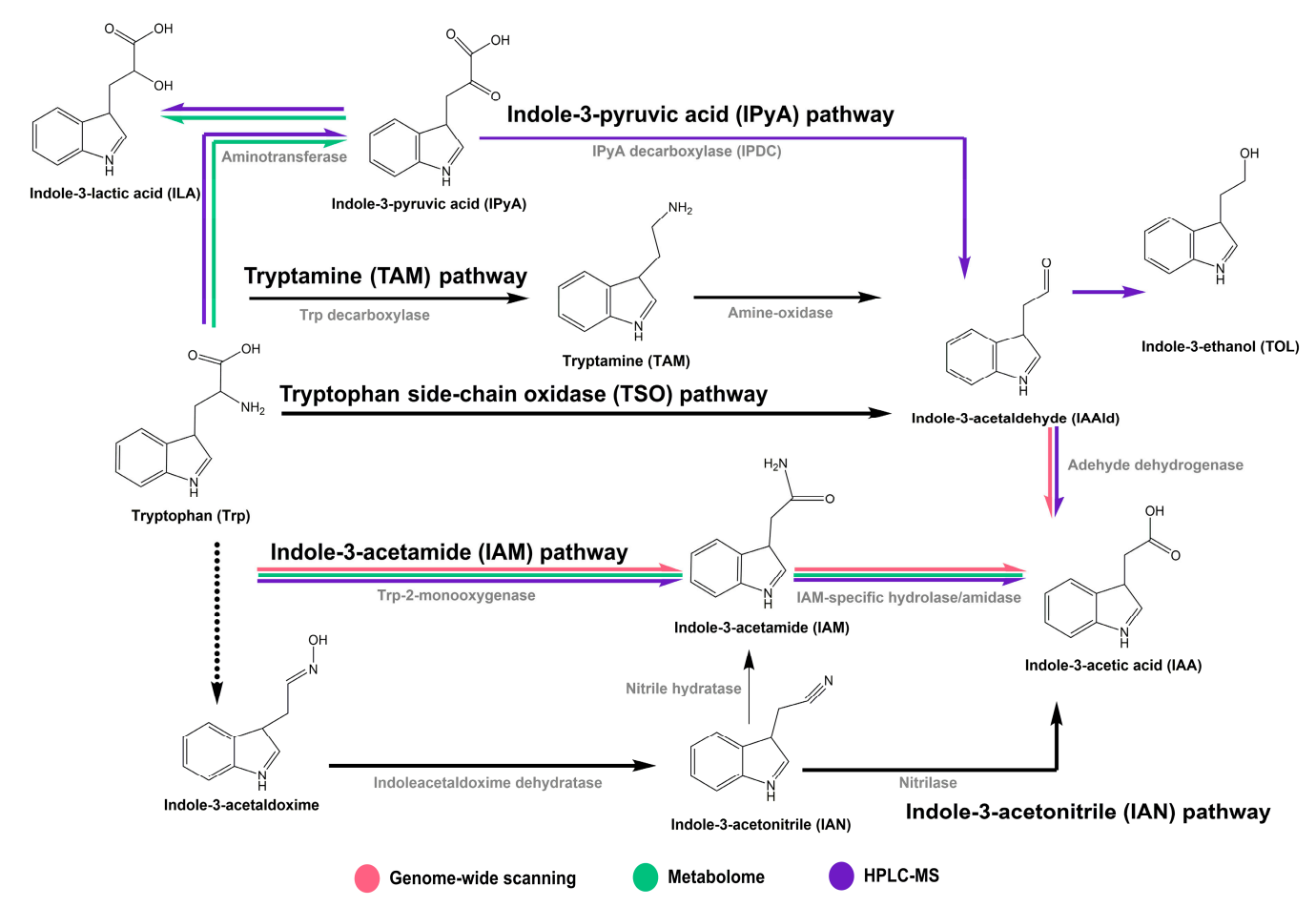

Figure 5. Tryptophan-dependent pathway for IAA biosynthesis in bacteria. IAM: indole-3-acetamide; IPyA: indole-3-pyruvic acid; IAAld: indole-3-acetaldehyde; TAM: tryptamine; IAN: indole-3-acetonitrile; ILA: indole-3-lactic acid; TOL: indole-3-ethanol. Red lines: the IAA biosynthesis pathways in ZZ21 detected by genome-wide analysis; green lines: the IAA biosynthesis pathways in ZZ21 detected by metabolomic analysis; purple lines: the IAA biosynthesis pathways in ZZ21 identified by HPLC-MS.

The presence of a particular IAA biosynthesis pathway in bacteria is often proposed based on the identification of the corresponding intermediates. IAM, IPyA, IAAld, TAM and IAN are the main intermediate products in tryptophan-dependent IAA biosynthesis $[4,8,19]$. According to our metabolomic analysis, only three indole compounds were detected among the metabolites of ZZ21, namely IAM, IPyA and ILA. The identification of IAM indicates that in Arthrobacter pascens ZZ21, IAA is synthesized by the IAM pathway. The intermediate IPyA and its enzymatic reduction product ILA were also found among the metabolites of ZZ21. Subsequently, using HPLC-MS, we demonstrated that ILA was present among the metabolites of ZZ21, and we also identified TOL, the enzymatic reduction product of IAAld. Taken together, these results suggest that the IAM and IPyA pathways are used by ZZ21 to synthesize IAA, even though the genes encoding aminotransferase and indole-3-pyruvate decarboxylase in the IPyA pathway were not detected in the ZZ21 genome 
(Figure S1), perhaps due to the limitations of genomic DNA fragmentation. Similarly, Mino reported that tryptophan is metabolized to IAA via IPyA and IAAld in Arthrobacter and that IAM also forms as a product of tryptophan oxidation in this genus [17]. In addition, a comparison of the relative intensities of intermediates involved in the IAM and IPyA pathways suggests that the IAM intermediate could accumulate much more easily than the intermediates IPyA and ILA in the IPyA pathway of ZZ21 (Figure 2). Interestingly, the IPyA pathway was much more sensitive to exogenous tryptophan than the IAM pathway in ZZ21 (Figures $1 \mathrm{~b}$ and 4), which may be due to the increased activity of the key enzyme encoded by ipdC and the increased expression of this gene after the addition of exogenous tryptophan. This hypothesis has been demonstrated in both Azospirillum brasilense Sp7 and Pseudomonas putida GR12-2 [35,54,55].

The failure to detect the TAM intermediate among the metabolites of ZZ21, combined with the results of the genomic analysis, strongly suggest that ZZ21 does not employ the TAM pathway to synthesize IAA. The TSO pathway has thus far only been found in Pseudomonas fluorescens CHA0 [41]. This observation, combined with the results of our genome-wide analysis, indicates that the TSO pathway is unlikely to exist in ZZ21. IAN was also not identified in ZZ21, which is consistent with the lack of genes encoding enzymes in the IAN pathway in the genome scanning experiment and suggests that IAA biosynthesis in Arthrobacter does not occur through the IAN pathway.

Since ZZ21 was able to produce IAA in the absence of an exogenous tryptophan supply (Figure 1a), we thought that the species might have the ability to synthesize tryptophan as an endogenous precursor or that a tryptophan-independent IAA biosynthesis pathway might function in ZZ21. The tryptophan-independent IAA biosynthesis pathway was first identified in Arabidopsis thaliana, as proposed by Normanly et al. [56,57]. The tryptophan-independent IAA biosynthesis pathway in bacteria was first demonstrated in Azospirillum brasilense using labeled tryptophan-feeding experiments [9]. However, no related enzymes in this pathway have thus far been identified. Thus, the existence of a tryptophan-independent pathway has not been confirmed and requires further study [58].

In conclusion, the results of this study indicate that the IAM and IPyA pathways function in tryptophan-dependent IAA biosynthesis in the plant-beneficial Arthrobacter pascens ZZ21 (Figure 5). Moreover, an uncharacterized tryptophan-independent pathway for IAA biosynthesis likely exists in A. pascens ZZ21.

\section{Materials and Methods}

\subsection{Chemicals and Materials}

L-tryptophan (Trp), indole-3-pyruvic acid (IPyA), indole-3-acetamide (IAM, 98\%), and indole3-ethanol (TOL, 97\%) were purchased from Sigma-Aldrich (St. Louis, MO, USA). Indole-3-lactic acid (ILA, >98\%) was purchased from Chemical Industry Co., Ltd. (Tokyo, Japan), and indole-3-acetic acid (IAA, $>99 \%$ ) was purchased from Aladdin (Shanghai, China). Methanol, pyridine, n-hexane, methoxylamine hydrochloride (97\%), and $\mathrm{N}, \mathrm{O}$-Bis (trimethylsilyl) trifluoroacetamide (BSTFA) with $1 \%$ trimethylchlorosilane (TMCS) were purchased from CNW Technologies GmbH (Düsseldorf, Germany). L-2-chlorophenylalanine was purchased from Shanghai Hengchuang Bio-technology Co., Ltd. (Shanghai, China). All chemicals and solvents were analytical or High-performance liquid chromatography (HPLC) grade.

\subsection{Bacterial Strain and Growth Conditions}

Arthrobacter pascens strain ZZ21 (China General Microbiology Culture Collection Center, CGMCC accession no. 7325) was grown at $30^{\circ} \mathrm{C}$ with shaking at $250 \mathrm{rpm}$ for $48 \mathrm{~h}$. Minimal liquid medium contained $5 \mathrm{~g} \cdot \mathrm{L}^{-1}$ glucose, $2 \mathrm{~g} \cdot \mathrm{L}^{-1}\left(\mathrm{NH}_{4}\right)_{2} \mathrm{SO}_{4}, 0.5 \mathrm{~g} \cdot \mathrm{L}^{-1} \mathrm{NaH}_{2} \mathrm{PO}_{4}, 0.5 \mathrm{~g} \cdot \mathrm{L}^{-1} \mathrm{~K}_{2} \mathrm{HPO}_{4}, 0.2 \mathrm{~g} \cdot \mathrm{L}^{-1}$ $\mathrm{MgSO}_{4} \cdot 7 \mathrm{H}_{2} \mathrm{O}$, and $0.1 \mathrm{~g} \cdot \mathrm{L}^{-1} \mathrm{CaCl}_{2} \cdot 2 \mathrm{H}_{2} \mathrm{O}$ at pH 7.0. Luria-Bertani (LB) medium contained $10 \mathrm{~g} \mathrm{NaCl}$, $10 \mathrm{~g}$ peptone, and $5 \mathrm{~g}$ yeast extract. When required, $200 \mathrm{mg} \cdot \mathrm{L}^{-1} \mathrm{~L}$-tryptophan was added to the medium to stimulate IAA production. 


\subsection{Quantification of IAA Levels}

IAA levels in the supernatants of $A$. pascens ZZ21 cultures were estimated spectrophotometrically according to the method of Gordon and Weber [59]. Each 2-mL aliquot of supernatant was combined with $2 \mathrm{~mL}$ Salkowski reagent $\left(50 \mathrm{~mL}\right.$ of $35 \% \mathrm{HClO}_{4}+1 \mathrm{~mL}$ of $0.5 \mathrm{M} \mathrm{FeCl}_{3}$ ) and incubated in darkness for $30 \mathrm{~min}$ at room temperature for color development. Absorbance was measured at $530 \mathrm{~nm}$ using a UV-VIS spectrophotometer (Uvmini-1240; Shimadzu, Japan). The IAA concentration was calculated by comparing the absorbance with a standard curve constructed using known concentrations of IAA.

\subsection{Analysis of Genes Involved in IAA Production}

Genomic DNA was extracted from A. pascens ZZ21, detected by 1\% agarose gel electrophoresis, and separated into 400-500 bp fragments with a Covaris M220 Focused Ultrasonicator. An Illumina paired-end (PE) library (460 bp library) was constructed for sequencing analysis, and the entire bacterial genome was subjected to bioinformatics analysis. Illumina MiSeq sequencing technology was used to sequence the genome of strain ZZ21. Genomic rRNA and tRNA were predicted using Barrnap 0.4.2 and tRNAscan-SE v1.3.1 software, respectively. Genes were predicted using Glimmer 3.02 software. Deduced protein sequences of the predicted genes were Blastp-aligned (blast 2.2.28+) with the $\mathrm{Nr}$, genes, string and GO databases to obtain annotation information for the predicted genes. The obtained predicted genes were blast-aligned (blastx/blastp 2.2.28+) with the KEGG database, and the specific biological pathway involved in the corresponding gene can be obtained according to the KEGG Orthology $(\mathrm{KO})$ number obtained from the alignment. The genes were identified based on their annotated information, which predicted putative enzyme activities already known to function in IAA metabolism.

\subsection{Quantitative Reverse-Transcription PCR Analysis of Genes Involved in IAA Biosynthesis}

For functional gene validation assays, cDNA (complementary DNA) was directly extracted from A. pascens ZZ21 in minimal liquid medium with or without $200 \mathrm{mg} \cdot \mathrm{L}^{-1}$ L-tryptophan using a CellAmp ${ }^{\mathrm{TM}}$ Whole Transcriptome Amplification Kit (Real Time) (TaKaRa, Dalian, China). The resulting cDNA was diluted 1:100 in RNase-free (Ribonuclease-free) water and used as template DNA for quantitative reverse-transcription PCR (qRT-PCR) with a SYBR Premix Ex Taq Kit (TaKaRa, Dalian, China). The reactions were carried out on an ABI Step One Plus Real-Time PCR system under the following conditions: $3 \mathrm{~min}$ at $95{ }^{\circ} \mathrm{C}$ for denaturation, followed by 40 cycles of $10 \mathrm{~s}$ at $95{ }^{\circ} \mathrm{C}$, $30 \mathrm{~s}$ at $55^{\circ} \mathrm{C}$, and $20 \mathrm{~s}$ at $72{ }^{\circ} \mathrm{C}$. The primers used for RT-PCR are listed in Table 2 . The $16 \mathrm{~s}$ rDNA (ribosomal DNA) gene from ZZ21 served as an internal control. The qRT-PCR data were analyzed using the $2^{-\Delta \Delta C t}$ method.

Table 2. Primers used in this study.

\begin{tabular}{|c|c|}
\hline Primer Name & Sequence $5^{\prime}-3^{\prime}$ a \\
\hline gatAF & CGAAACCACCATCCGCTACG \\
\hline gatAR & TGGAACTGGCGAAGAAAGGC \\
\hline iaaMF & CCTGGAAAGCCGCTGTGA \\
\hline iaaMR & GCCGTAGAAGGTCTGCTCGTC \\
\hline prrF & ACTTCGGTCCGCTGAACAAC \\
\hline prrR & CATCTCCACGGCTTCCTGTT \\
\hline puuCF & GCCGCAGCAATCTCAAGC \\
\hline puuCR & CCAGCAACGCAGCGAAGT \\
\hline aamF & GCAACATTGTCGGCTTCAGG \\
\hline aamR & CGGACCGAAAGACCTGGG \\
\hline aldHF & GCAACACGGTGGTCTGGAAG \\
\hline aldHR & GCCCAACAGGAACGGAACC \\
\hline $16 \mathrm{sF}$ & GGTTGCGATACTGTGAGGTG \\
\hline $16 s R$ & CTCCCACAAGGGTTAGGC \\
\hline
\end{tabular}




\subsection{Metabolomic Analysis of the IAA Biosynthesis Pathway in ZZ21}

After $48 \mathrm{~h}$ of culture in minimal medium, the fermentation broth of A. pascens ZZ21 was collected by gravity, filtered through a $0.22 \mu \mathrm{m}$ filter, lyophilized, and stored at $-80{ }^{\circ} \mathrm{C}$. The lyophilized metabolites were re-dissolved in $10 \mathrm{~mL}$ methanol-water $(4: 1, v / v)$, and $20 \mu \mathrm{L}$ of internal standard (L-2-chloro-phenylalanine, $0.3 \mathrm{mg} \cdot \mathrm{mL}^{-1}$ in methanol) was added to $1 \mathrm{~mL}$ of each sample solution. After centrifugation for $10 \mathrm{~min}\left(12,000 \mathrm{rpm}, 4^{\circ} \mathrm{C}\right), 200 \mu \mathrm{L}$ of the supernatant was loaded into a glass vial and lyophilized in a freeze concentration centrifugal dryer (LNG-T98, Taicang, China). After adding $80 \mu \mathrm{L}$ methoxyamine hydrochloride pyridine solution $\left(15 \mathrm{mg} \cdot \mathrm{mL}^{-1}\right)$ to the vial, the sample was vortexed for $2 \mathrm{~min}$ before being subjected to an oximation reaction in a shaking incubator at $37^{\circ} \mathrm{C}$ for $90 \mathrm{~min}$. After incubation, the sample was combined with $80 \mu \mathrm{L}$ of BSTFA (1\% TMCS) derivatization reagent and $20 \mu \mathrm{L}$ of n-hexane. After vortexing for $2 \mathrm{~min}$, the sample was reacted at $70{ }^{\circ} \mathrm{C}$ for $60 \mathrm{~min}$ and allowed to stand for $30 \mathrm{~min}$ at room temperature prior to Gas Chromatography-mass Spectrometer (GC-MS) metabolomics analysis. Quality control samples $(\mathrm{QC})$ were prepared by mixing equal volumes of extracts from all samples, each at the same volume as the sample.

An Agilent 7890B gas chromatography system coupled to an Agilent 5977A MSD system (Agilent Technologies Inc., Santa Clara, CA, USA) was used to analyze the derivatized samples. The derivatives were separated by a DB-5MS fused-silica capillary column $(30 \mathrm{~m} \times 0.25 \mathrm{~mm} \times 0.25 \mu \mathrm{m}$, Agilent J \& W Scientific, Folsom, CA, USA). Helium ( $>99.999 \%$ ) was used as carrier gas, with a constant flow rate of $1 \mathrm{~mL} / \mathrm{min}$ through the column. The injector temperature was maintained at $260{ }^{\circ} \mathrm{C}$. An injection volume of $1 \mu \mathrm{L}$ was used for split mode injection (split ratio 10:1). The initial oven temperature was $60^{\circ} \mathrm{C}$, which was ramped up to $125^{\circ} \mathrm{C}$ at a rate of $8{ }^{\circ} \mathrm{C} / \mathrm{min}$, followed by ramping up to $210{ }^{\circ} \mathrm{C}$ at $5{ }^{\circ} \mathrm{C} / \mathrm{min}$, to $270{ }^{\circ} \mathrm{C}$ at $10^{\circ} \mathrm{C} / \mathrm{min}$, and to $305^{\circ} \mathrm{C}$ at $20^{\circ} \mathrm{C} / \mathrm{min}$ and was held at $305^{\circ} \mathrm{C}$ for $5 \mathrm{~min}$. The temperature of the MS quadrupole and ion source (electron impact) was set to 150 and $230{ }^{\circ} \mathrm{C}$, respectively. The collision energy was $70 \mathrm{eV}$. Mass spectrometric data were acquired in full-scan mode $(m / z 50-450)$, and the solvent delay time was set to $5 \mathrm{~min}$. A QC sample was inserted after every 10 analytical samples to assess the reproducibility of the entire analysis.

ChemStation (version E.02.02.1431, Agilent, Santa Clara, CA, USA) was used to convert the file format of raw data from the apparatus to common data format (CDF). ChromaTOF (version 4.34, LECO, St. Joseph, MI, USA) was used to analyze the data. Metabolites were subjected to qualitative analysis by National Institute of Standards and Technology (NIST) and Fiehn database.

\subsection{Identification of the Intermediates in the IAA Biosynthesis Pathway of ZZ21 by HPLC-MS}

Indole derivatives were extracted from culture supernatants of $A$. pascens ZZ21 grown for $48 \mathrm{~h}$ in minimal medium supplied with $200 \mathrm{mg} \cdot \mathrm{L}^{-1} \mathrm{~L}$-tryptophan at $30{ }^{\circ} \mathrm{C}$ by centrifugation at $8000 \mathrm{rpm}$ for $10 \mathrm{~min}$. The culture supernatants were acidified to $\mathrm{pH} 2.5$ using $1 \mathrm{M} \mathrm{HCl}$ and extracted twice using a double volume of ethyl acetate. A fraction of ethyl acetate was air dried and re-dissolved in one-tenth volume of methanol [60]. These methanolic extracts were subjected to HPLC-MS/MS analysis.

An Agilent Technologies 1200 Series RRLC system coupled with an Agilent 6410 triple-quadrupole mass spectrometer (Agilent Technologies Inc., Santa Clara, CA, USA) equipped with an electrospray ion source (ESI) and operated in positive ion mode was used for analysis. An Agilent ZORBAX Eclipse Plus C18 column $(2.1 \mathrm{~mm} \times 150 \mathrm{~mm}, 3.5 \mu \mathrm{m})$ (Agilent Technologies Inc., Santa Clara, CA, USA) with mobile phases A (methanol) and B (0.8\% formic acid in water) $(6: 4, v / v)$ was used, with the column temperature set to $30^{\circ} \mathrm{C}$ and a flow rate of $0.2 \mathrm{~mL} / \mathrm{min}$. The total run time was $10 \mathrm{~min}$, and the injection volume was $0.2 \mu \mathrm{L}$. The positive ionization mode was used at a spray voltage of $4000 \mathrm{~V}$. The nebulizer gas $\left(\mathrm{N}_{2}\right)$ pressure was set to 30 psi with a flow rate of $10 \mathrm{~L} / \mathrm{min}$. The heated capillary temperature was $330^{\circ} \mathrm{C}$. Mass spectrometric scanning was performed from 50 to 500 mass. The molecular weight of IAA, IAM, ILA and TOL standards are 175.1, 174.2, 205.1 and 161.2, and after hydrogenation, the relative molecular masses of the four indole compounds are 176.1, 175.2, 206.1, and 162.2, respectively. The fragmentor voltage was $135 \mathrm{~V}$ for mass spectrometry analysis and $90 \mathrm{~V}$ for secondary ion mass spectrometry analysis. 


\subsection{Statistical Analyses}

One-way analysis of variance (ANOVA) was performed, with a $p$-value of $<0.05$ considered to be statistically significant. All statistical analyses were conducted using SPSS 22.0.

Supplementary Materials: The following are available online at www.mdpi.com/1422-0067/19/2/443/s1.

Acknowledgments: This study was supported by the National Natural Science Foundation of China (No. 41571244), Jiangsu Agriculture Science and Technology Innovation Fund (CX(17)1101) and the China Postdoctoral Science Foundation (No. 2016M600421). We thank Kathleen Farquharson and Jennifer Lockhart for the language improvement and valuable comments.

Author Contributions: Huixin Li, Jun Wu and Mengsha Li conceived and designed the experiments; Mengsha Li, Rui Guo, Fei Yu and Haiyan Zhao performed the experiments; Mengsha Li and Xu Chen analyzed the data; Mengsha Li, Huixin Li and Jun Wu contributed reagents/materials/analysis tools; Mengsha Li and Huixin Li wrote paper.

Conflicts of Interest: The authors declare no conflicts of interest.

\section{Abbreviations}

$\begin{array}{ll}\text { IAA } & \text { Indole-3-aectic acid } \\ \text { IAM } & \text { Indole-3-acetamide } \\ \text { IPyA } & \text { Indole-3-pyruvic acid } \\ \text { IAN } & \text { Indole-3-acetonitrile } \\ \text { ILA } & \text { Indole-3-lactic acid } \\ \text { IAAld } & \text { Indole-3-acetaldehyde } \\ \text { TOL } & \text { Indole-3-ethanol } \\ \text { TSO } & \text { Tryptophan side-chain oxidase } \\ \text { TAM } & \text { Tryptamine } \\ \text { IPDC } & \text { Indole-3-pyruvate decarboxylase } \\ \text { Trp } & \text { Tryptophan } \\ \text { TIC } & \text { Total ion current } \\ \text { EIC } & \text { Extracted ion chromatography } \\ \text { HPLC-MS } & \text { High-performance liquid chromatography-mass spectrometry } \\ \text { qRT-PCR } & \text { Quantitative reverse-transcription PCR } \\ \text { MRM } & \text { Multiple reaction monitoring } \\ \text { KEGG } & \text { Kyoto Encyclopedia of Genes and Genomes } \\ \text { NCBI } & \text { National Center for Biotechnology Information } \\ \text { LB } & \text { Luria-Bertani } \\ \text { CK } & \text { Control check } \\ \text { KO } & \text { KEGG Orthology } \\ \text { NIST } & \text { National Institute of Standards and Technology } \\ & \end{array}$

\section{References}

1. Woodward, A.W.; Bartel, B. Auxin: Regulation, action, and interaction. Ann. Bot. 2005, 95, 707-735. [CrossRef] [PubMed]

2. Teale, W.D.; Paponov, I.A.; Palme, K. Auxin in action: Signalling, transport and the control of plant growth and development. Nat. Rev. Mol. Cell Biol. 2006, 7, 847-859. [CrossRef] [PubMed]

3. Halliday, K.J.; Martínez-García, J.F.; Josse, E.M. Integration of light and auxin signaling. Cold Spring Harb. Perspect. Biol. 2009, 1, A001586. [CrossRef] [PubMed]

4. Spaepen, S.; Vanderleyden, J.; Remans, R. Indole-3-acetic acid in microbial and microorganism-plant signaling. FEMS Microbiol. Rev. 2007, 31, 425-448. [CrossRef] [PubMed]

5. Patten, C.L.; Glick, B.R. Bacterial biosynthesis of indole-3-acetic acid. Can. J. Microbiol. 1996, 42, $207-220$. [CrossRef] [PubMed]

6. Sessitsch, A.; Reiter, B.; Berg, G. Endophytic bacterial communities of field-grown potato plants and their plant-growth-promoting and antagonistic abilities. Can. J. Microbiol. 2004, 50, 239-249. [CrossRef] [PubMed] 
7. Carreño-Lopez, R.; Campos-Reales, N.; Elmerich, C.; Baca, B.E. Physiological evidence for differently regulated tryptophan-dependent pathways for indole-3-acetic acid synthesis in Azospirillum brasilense. Mol. Gen. Genet. 2000, 264, 521-530. [CrossRef] [PubMed]

8. Duca, D.; Lorv, J.; Patten, C.L.; Rose, D.; Glick, B.R. Indole-3-acetic acid in plant-microbe interactions. Antonie Van Leeuwenhoek 2014, 106, 85-125. [CrossRef] [PubMed]

9. Prinsen, E.; Costacurta, A.; Michiels, K.; Vanderleyden, J.; Van Onckelen, H. Azospirillum brasilense indole-3-acetic acid biosynthesis: Evidence for a non-tryptophan dependent pathway. Mol. Plant Microbe Interact. 1993, 6, 609. [CrossRef]

10. Phi, Q.T.; Park, Y.M.; Ryu, C.M.; Park, S.H.; Ghim, S.Y. Functional identification and expression of indole-3-pyruvate decarboxylase from Paenibacillus polymyxa E681. J. Microbiol. Biotechnol. 2008, 18, 1235-1244. [PubMed]

11. Vandeputte, O.; Öden, S.; Mol, A.; Vereecke, D.; Goethals, K.; El Jaziri, M.; Prinsen, E. Biosynthesis of auxin by the gram-positive phytopathogen Rhodococcus fascians is controlled by compounds specific to infected plant tissues. Appl. Environ. Microbiol. 2005, 71, 1169-1177. [CrossRef] [PubMed]

12. Shao, J.; Li, S.; Zhang, N.; Cui, X.; Zhou, X.; Zhang, G.; Zhang, R. Analysis and cloning of the synthetic pathway of the phytohormone indole-3-acetic acid in the plant-beneficial Bacillus amyloliquefaciens SQR9. Microb. Cell Factories 2015, 14, 130. [CrossRef] [PubMed]

13. Katznelson, H.; Sirois, J.C. Auxin production by species of Arthrobacter. Nature 1961, 191, $1323-1324$. [CrossRef] [PubMed]

14. Forni, C.; Riov, J.; Grilli, M.G.; Tel-Or, E. Indole-3-acetic acid (IAA) production by Arthrobacter species isolated from Azolla. Microbiology 1992, 138, 377-381. [CrossRef] [PubMed]

15. Yadav, A.N.; Sachan, S.G.; Verma, P.; Tyagi, S.P.; Kaushik, R.; Saxena, A.K. Culturable diversity and functional annotation of psychrotrophic bacteria from cold desert of Leh Ladakh (India). World J. Microbiol. Biotechnol. 2015, 31, 95-108. [CrossRef] [PubMed]

16. Ozdal, M.; Ozdal, O.G.; Sezen, A.; Algur, O.F.; Kurbanoglu, E.B. Continuous production of indole-3-acetic acid by immobilized cells of Arthrobacter agilis. Biotech 2017, 7, 23. [CrossRef] [PubMed]

17. Mino, Y. Studies on the Destruction of Indole-3-acetic Acid by a Species of Arthrobacter. V. Indole-3-acetic Acid Production from Tryptophan. Physiol. Plant. 1970, 23, 971-980. [CrossRef]

18. Zhang, Z.; Li, H.; Chen, X.; Li, W.M.; Li, F.H.; Xu, L. Isolation and characterization and fluoranthene degrading of an IAA secreting bacterial strain. Chin. J. Environ. Eng. 2014, 8, 5041-5048.

19. Zhang, Z. IAA-Secreting Bacteria, Flu-Degrading Bacteria Enhanced the Remediation of Flu in Soil by Plant; Nanjing Agricultural University: Nanjing, China, 2014.

20. Patten, C.L.; Blakney, A.J.; Coulson, T.J. Activity, distribution and function of indole-3-acetic acid biosynthetic pathways in bacteria. Crit. Rev. Microbiol. 2013, 39, 395-415. [CrossRef] [PubMed]

21. Ernstsen, A.; Sandberg, G.; Crozier, A.; Wheeler, C.T. Endogenous indoles and the biosynthesis and metabolism of indole-3-acetic acid in cultures of Rhizobium phaseoli. Planta 1987, 171, 422-428. [CrossRef] [PubMed]

22. Morris, R.O. Genes specifying auxin and cytokinin biosynthesis in prokaryotes. In Plant Hormones; Springer: Dordrecht, The Netherlands, 1995; pp. 318-399.

23. Sekine, M.; Watanabe, K.; Syono, K. Molecular cloning of a gene for indole-3-acetamide hydrolase from Bradyrhizobium japonicum. J. Bacteriol. 1989, 171, 1718-1724. [CrossRef] [PubMed]

24. Comai, L.; Kosuge, T. Cloning characterization of iaaM, a virulence determinant of Pseudomonas savastanoi. J. Bacteriol. 1982, 149, 40-46. [PubMed]

25. Clark, E.; Manulis, S.; Ophir, Y.; Barash, I.; Gafni, Y. Cloning and characterization of iaaM and iaaH from Erwinia herbicola pathovar gypsophilae. Phytopathology 1993, 83, 234-240. [CrossRef]

26. Asano, Y.; Tachibana, M.; Tani, Y.; Yamada, H. Purification and characterization of amidase which participates in nitrile degradation. Agric. Biol. Chem. 1982, 46, 1175-1181.

27. Kuo, T.T.; Kosuge, T. Factors influencing the production and further metabolism of indole-3-acetic acid by Pseudomonas savastanoi. J. Gen. Appl. Microbiol. 1969, 15, 51-63. [CrossRef]

28. Gaffney, T.D.; e Silva, O.D.C.; Yamada, T.; Kosuge, T. Indoleacetic acid operon of Pseudomonas syringae subsp. savastanoi: Transcription analysis and promoter identification. J. Bacteriol. 1990, 172, 5593-5601. [CrossRef] [PubMed] 
29. Hutcheson, S.W.; Kosuge, T. Regulation of 3-indoleacetic acid production in Pseudomonas syringae pv. savastanoi. Purification and properties of tryptophan 2-monooxygenase. J. Biol. Chem. 1985, 260, 6281-6287. [PubMed]

30. Kittell, B.L.; Helinski, D.R.; Ditta, G.S. Aromatic aminotransferase activity and indoleacetic acid production in Rhizobium meliloti. J. Bacteriol. 1989, 171, 5458-5466. [CrossRef] [PubMed]

31. Koga, J.; Syono, K.; Ichikawa, T.; Adachi, T. Involvement of L-tryptophan aminotransferase in indole-3-acetic acid biosynthesis in Enterobacter cloacae. Biochim. Biophys. Acta 1994, 1209, 241-247. [CrossRef]

32. Koga, J.; Adachi, T.; Hidaka, H. Molecular cloning of the gene for indolepyruvate decarboxylase from Enterobacter cloacae. Mol. Gen. Genet. 1991, 226, 10-16. [CrossRef] [PubMed]

33. Costacurta, A.; Keijers, V.; Vanderleyden, J. Molecular cloning and sequence analysis of an Azospirillum brasilense indole-3-pyruvate decarboxylase gene. Mol. Gen. Genet. 1994, 243, 463-472. [PubMed]

34. Brandl, M.T.; Lindow, S.E. Cloning and characterization of a locus encoding an indolepyruvate decarboxylase involved in indole-3-acetic acid synthesis in Erwinia herbicola. Appl. Environ. Microbiol. 1996, 62, 4121-4128. [PubMed]

35. Patten, C.L.; Glick, B.R. Role of Pseudomonas putida Indoleacetic Acid in Development of the Host Plant Root System. Appl. Environ. Microbiol. 2002, 68, 3795-3801. [CrossRef] [PubMed]

36. Pollmann, S.; Müller, A.; Weiler, E.W. Many roads lead to "auxin": Of nitrilases, synthases, and amidases. Plant Biol. 2006, 8, 326-333. [CrossRef] [PubMed]

37. Rajagopal, R. Metabolism of Indole-3-acetaldehyde. III. Some Characteristics of the Aldehyde Oxidase of Avena Coleoptiles. Physiol. Plant. 1971, 24, 272-281. [CrossRef]

38. Idris, E.E.; Iglesias, D.J.; Talon, M.; Borriss, R. Tryptophan-dependent production of indole-3-acetic acid (IAA) affects level of plant growth promotion by Bacillus amyloliquefaciens FZB42. Mol. Plant-Microbe Interact. 2007, 20, 619-626. [CrossRef] [PubMed]

39. Hartmann, A.; Singh, M.; Klingmüller, W. Isolation and characterization of Azospirillum mutants excreting high amounts of indoleacetic acid. Can. J. Microbiol. 1983, 29, 916-923. [CrossRef]

40. Perley, J.E.; Stowe, B.B. On the ability of Taphrina deformans to produce indoleacetic acid from tryptophan by way of tryptamine. Plant Physiol. 1966, 41, 234-237. [CrossRef] [PubMed]

41. Oberhänsli, T.; Dfago, G.; Haas, D. Indole-3-acetic acid (IAA) synthesis in the biocontrol strain CHA0 of Pseudomonas fluorescens: Role of tryptophan side chain oxidase. Microbiology 1991, 137, 2273-2279. [CrossRef] [PubMed]

42. Nelson, K.E.; Weinel, C.; Paulsen, I.T.; Dodson, R.J.; Hilbert, H.; Martins dos Santos, V.A.P.; Brinkac, L. Complete genome sequence and comparative analysis of the metabolically versatile Pseudomonas putida KT2440. Environ. Microbiol. 2002, 4, 799-808. [CrossRef] [PubMed]

43. Jo, J.E.; Raj, S.M.; Rathnasingh, C.; Selvakumar, E.; Jung, W.C.; Park, S. Cloning, expression, and characterization of an aldehyde dehydrogenase from Escherichia coli $\mathrm{K}-12$ that utilizes 3-Hydroxypropionaldehyde as a substrate. Appl. Microbiol. Biotechnol. 2008, 81, 51-60. [CrossRef] [PubMed]

44. Turroni, F.; Bottacini, F.; Foroni, E.; Mulder, I.; Kim, J.H.; Zomer, A.; Delledonne, M. Genome analysis of Bifidobacterium bifidum PRL2010 reveals metabolic pathways for host-derived glycan foraging. Proc. Natl. Acad. Sci. USA 2010, 107, 19514-19519. [CrossRef] [PubMed]

45. Moreno, B.; Nogales, R.; Sánchez, L.; Benítez, E. Re-start of olive waste vermicompost through addition of tryptophan and its effects on indole-3-acetic acid in pepper rhizosphere when used as soil amendment. Sci. Hortic. 2017, 221, 16-22. [CrossRef]

46. Lindahl, R. Aldehyde dehydrogenases and their role in carcinogenesis. Crit. Rev. Biochem. Mol. Biol. 1992, 27, 283-335. [CrossRef] [PubMed]

47. Perozich, J.; Nicholas, H.; Wang, B.C.; Lindahl, R.; Hempel, J. Relationships within the aldehyde dehydrogenase extended, family. Protein Sci. 1999, 8, 137-146. [CrossRef] [PubMed]

48. Hazelwood, L.A.; Daran, J.M.; van Maris, A.J.; Pronk, J.T.; Dickinson, J.R. The Ehrlich pathway for fusel alcohol production: A century of research on Saccharomyces cerevisiae metabolism. Appl. Environ. Microbiol. 2008, 74, 2259-2266. [CrossRef] [PubMed]

49. Kato, Y.; Yoshida, S.; Asano, Y. Polymerase chain reaction for identification of aldoxime dehydratase in aldoxime- or nitrile-degrading microorganisms. FEMS Microbiol. Lett. 2005, 246, 243-249. [CrossRef] [PubMed] 
50. Bartling, D.; Seedorf, M.; Mithöfer, A.; Weiler, E.W. Cloning and expression of an Arabidopsis, nitrilase which can convert indole-3-acetonitrile to the plant hormone, indole-3-acetic acid. FEBS J. 1992, 205, 417-424. [CrossRef]

51. Zhao, Y. Auxin Biosynthesis: A Simple Two-Step Pathway Converts Tryptophan to Indole-3-Acetic Acid in Plants. Mol. Plant 2012, 5, 334-338. [CrossRef] [PubMed]

52. Asano, Y.; Fujishiro, K.; Tani, Y.; Yamada, H. Aliphatic Nitrile Hydratase from Arthrobacter sp. J-1 Purification and Characterization. Agric. Biol. Chem. 1982, 46, 1165-1174. [CrossRef]

53. Bandyopadhyay, A.K.; Nagasawa, T.; Asano, Y.; Fujishiro, K.; Tani, Y.; Yamada, H. Purification and Characterization of Benzonitrilases from Arthrobacter sp. Strain J-1. Appl. Environ. Microbiol. 1986, 51, 302-306. [PubMed]

54. Zimmer, W.; Wesche, M.; Timmermans, L. Identification and isolation of the indole-3-pyruvate decarboxylase gene from Azospirillum brasilense Sp7: Sequencing and functional analysis of the gene locus. Curr. Microbiol. 1998, 36, 327-331. [CrossRef] [PubMed]

55. Patten, C.L.; Glick, B.R. Regulation of indoleacetic acid production in Pseudomonas putida GR12-2 by tryptophan and the stationary-phase sigma factor RpoS. Can. J. Microbiol. 2002, 48, 635-642. [CrossRef] [PubMed]

56. Last, R.L.; Bissinger, P.H.; Mahoney, D.J.; Radwanski, E.R.; Fink, G.R. Tryptophan mutants in Arabidopsis: The consequences of duplicated tryptophan synthase beta genes. Plant Cell 1991, 3, 345-358. [CrossRef] [PubMed]

57. Normanly, J.; Cohen, J.D.; Fink, G.R. Arabidopsis thaliana auxotrophs reveal a tryptophan-independent biosynthetic pathway for indole-3-acetic acid. Proc. Natl. Acad. Sci. USA 1993, 90, 10355-10359. [CrossRef] [PubMed]

58. Müller, A.; Weiler, E.W. Indolic constituents and indole-3-acetic acid biosynthesis in the wild-type and a tryptophan auxotroph mutant of Arabidopsis thaliana. Planta 2000, 211, 855-863. [CrossRef] [PubMed]

59. Gordon, S.A.; Weber, R.P. Colorimetric estimation of indoleacetic acid. Plant Physiol. 1951, 26, 192. [CrossRef] [PubMed]

60. Goswami, D.; Dhandhukia, P.; Patel, P.; Thakker, J.N. Screening of PGPR from saline desert of Kutch: Growth promotion in Arachis hypogea by Bacillus licheniformis A2. Microbiol. Res. 2014, 169, 66-75. [CrossRef] [PubMed] 